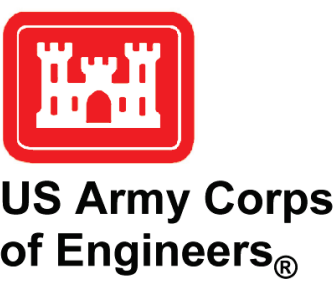

\title{
AIS Data Case Study: Identifying AIS Coverage Gaps on the Ohio River in CY2018
}

\section{by Patricia K. DiJoseph, Brian J. Tetreault, Marin M. Kress}

PURPOSE: This Coastal and Hydraulics Engineering Technical Note (CHETN) describes a method for evaluating the received coverage from Automatic Identification System (AIS) shore sites and the availability of historic vessel position reports along the Ohio River. The network of AIS shoreside sites installed and operated by the US Army Corps of Engineers (USACE) and the US Coast Guard (USCG) receive information transmitted from vessels; however, reception of these transmissions is generally line-of-sight between the vessel and the AIS site antenna. Reception may also be affected by factors such as the quality of the transceiver installation aboard the vessel as well as the state of the equipment at the receiving site. Understanding how to define and quantify coverage gaps along the inland river system can inform research utilizing AIS data, provide information on the performance of the AIS network, and provide guidance for efforts to address identified coverage gaps.

INTRODUCTION: The ability to access historical vessel position reports through the USCG Nationwide Automatic Identification System (NAIS) archive (USCG 2018) has expanded the tools available for commercial vessel waterway utilization studies (DiJoseph et al. 2019; Mitchell and Scully 2014; Scully and Mitchell 2015) and provided waterways managers with a valuable tool for understanding local navigation traffic and related dredging needs (Kress et al. 2020; Mitchell et al. 2020; Tabbert et al. 2020). AIS is also used for transmitting real-time electronic aids to navigation for navigational safety and security. Since 2016, AIS carriage requirements include most commercial self-propelled vessels on US navigable waters including any vessel over $65 \mathrm{ft}^{1,2}$ in length, towing vessels over $26 \mathrm{ft}$ in length with a greater than $600 \mathrm{hp}$ engine, vessels certified to carry 150 or more passengers, dredges in or near a commercial channel, and vessels moving certain dangerous cargo (USCG 2018; US Code of Regulations 2019).

In general, AIS messages are transmitted from vessels and received by both nearby vessels and shoreside AIS sites (shoreside AIS sites may also broadcast messages, but this paper focuses on vessel position reports as received by shoreside AIS sites). The messages received by shore AIS sites are routed to the USCG, where they are archived. AIS operates using the very high frequency (VHF) radio spectrum, operating on $161.975 \mathrm{MHz}$ and $162.025 \mathrm{MHz}$. VHF transmissions are generally lineof-sight (i.e., the antenna of the transmitting site must have an unobstructed path to the receiving site's antenna). The transmission may be attenuated or blocked by the earth (radio horizon based on antenna

\footnotetext{
${ }^{1}$ For a full list of the spelled-out forms of the units of measure used in this document, please refer to US Government Publishing Office Style Manual, 31 ${ }^{\text {st }}$ ed. (Washington, DC: US Government Publishing Office, 2016), 248-52, https://www.govinfo.gov/content/pkg/GPO-STYLEMANUAL-2016/pdf/GPO-STYLEMANUAL-2016.pdf

${ }^{2}$ For a full list of the unit conversions used in this document, please refer to US Government Publishing Office Style Manual, 31st ed. (Washington, DC: US Government Publishing Office 2016), 345-7, https://www.govinfo.gov/content/pkg/GPO-STYLEMANUAL-2016/pdf/GPO-STYLEMANUAL-2016.pdf.
} 
heights, or terrain) or man-made structures. The signal may also be reduced by other factors, such as transmission power, equipment condition, and quality of installation. Therefore, not all AIS signals transmitted by vessels are received by shore AIS sites. AIS technical standards and history can be found online through international associations involved in setting technical standards (IALA 2008; IEC 2001; ITU-R 2014; PIANC 2019). Future studies are planned to measure how different factors affect AIS site reception rate.

Vessels transmit AIS position reports at different time intervals depending on their dynamic condition; the reporting intervals are shown in Table 1. Thus, for the purposes of understanding if sufficient data are available for historical analysis, one measure of AIS coverage is the ability to recreate a historic vessel trackline with fidelity at a specific time interval, such as a $5 \mathrm{~min}, 1 \mathrm{~min}, 30 \mathrm{~s}$, or $10 \mathrm{~s}$ level (this is a different definition of coverage than may be used for other purposes, such as for waterway monitoring for safety or security).

\begin{tabular}{|l|l||}
\hline \multicolumn{2}{|l||}{ Table 1. Class A shipborne mobile equipment reporting intervals. } \\
\hline \hline Ship dynamic conditions & Nominal reporting interval \\
\hline Ship at anchor or moored and not moving faster than $3 \mathrm{kn}$ & $3 \mathrm{~min}$ \\
\hline Ship at anchor or moored and moving faster than $3 \mathrm{kn}$ & $10 \mathrm{~s}$ \\
\hline Ship 0-14 kn & $10 \mathrm{~s}$ \\
\hline Ship 0-14 kn and changing course & $31 / 3 \mathrm{~s}$ \\
\hline Ship 14-23 kn & $6 \mathrm{~s}$ \\
\hline Ship 14-23 kn and changing course & $2 \mathrm{~s}$ \\
\hline Ship >23 kn & $2 \mathrm{~s}$ \\
\hline Ship >23 kn and changing course & $2 \mathrm{~s}$ \\
\hline \hline
\end{tabular}

Source: ITU-R M.1371-5, 2014, page 8.

This study examined the availability of AIS data for the entirety of the Ohio River, from River Mile 0 , located at the confluence of the Ohio, Allegheny, and Monongahela Rivers to River Mile 981, located at the confluence of the Ohio, Upper Mississippi, and Lower Mississippi Rivers, as shown in Figure 1. This is a heavily traveled portion of US inland waterways; in 2018, the Ohio River carried over 178 million short tons of commodities in the span from Pittsburgh, PA, to the confluence with the Mississippi River (WCSC 2021). Table 2 lists the AIS sites along the waterway installed and operated by both the USCG through the NAIS, and USACE through the Lock Operations Management Application (LOMA). NAIS has four sites along the Ohio River at Pittsburgh, PA; Huntington, WV; Louisville, KY; and Cincinnati, OH (Fort Thomas). USACE has multiple sites located at locks along the Ohio River and various tributaries. Figure 1 shows the locations of LOMA AIS sites, Ohio River navigation locks, and the Ohio River study area (dark blue). The terrain around the Ohio River is quite rolling, and the locks are located in river valleys where surrounding hills may interrupt AIS signal transmission. Because of the combination of terrain and distance between sites, there are coverage gaps between many of these sites. 


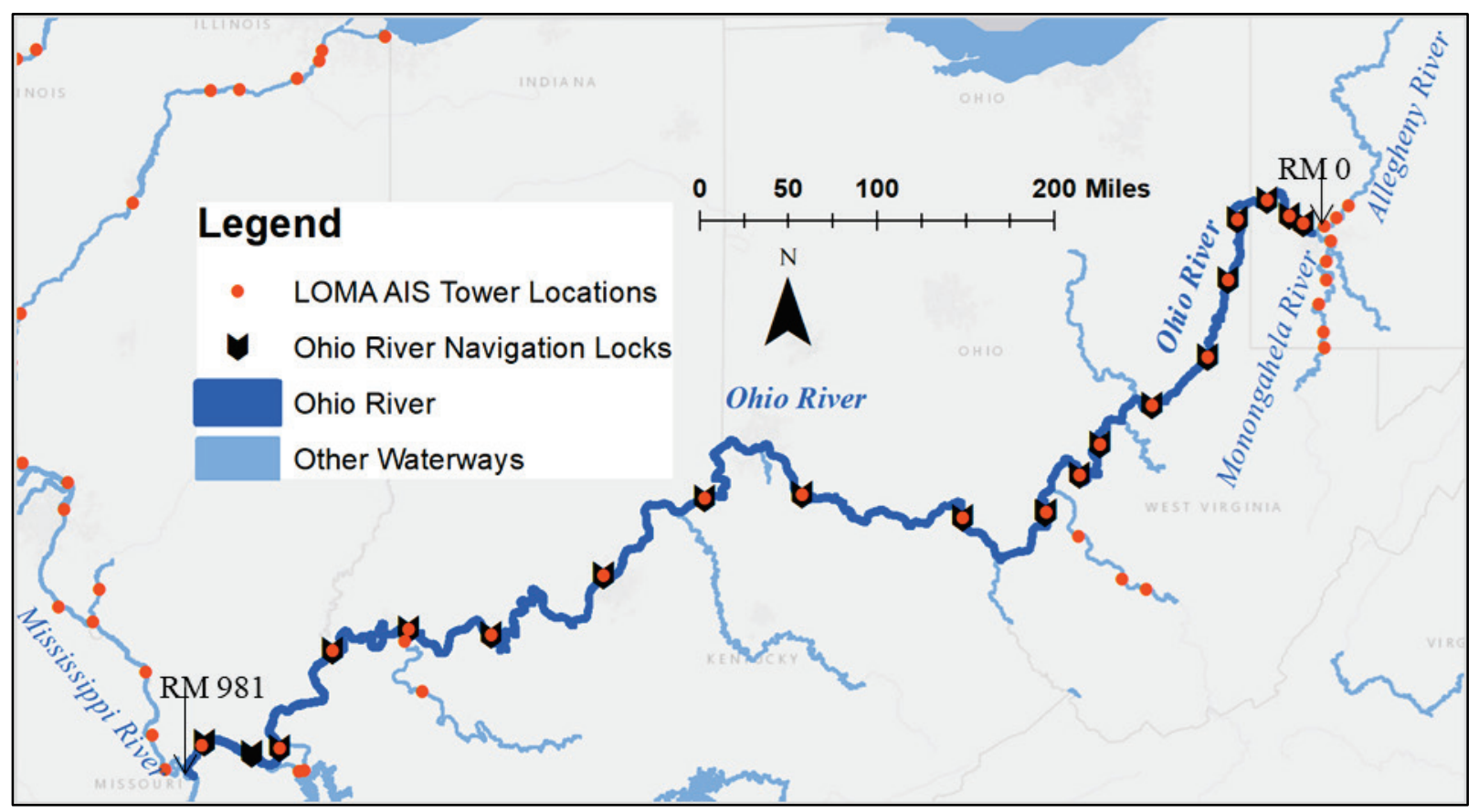

Figure 1. Map of Ohio River (dark blue), navigation locks (black arrows), and USACE LOMA AIS shoreside AIS sites (orange dots) along both the Ohio River and tributaries.

\begin{tabular}{|c|c|c|c|c|c|}
\hline Site Name & $\begin{array}{l}\text { Antenna } \\
\text { Height above } \\
\text { Ground (ft) }\end{array}$ & Latitude & Longitude & River & RM \\
\hline Prices Landing & 20 & 37.02594 & -89.35644 & Mississippi & 29.5 \\
\hline Olmsted L\&D** & 75 & 37.1835883 & -89.063833 & Ohio & 964.6 \\
\hline Barkley L\&D & 25 & 37.0192 & -88.22498 & Cumberland & 30.6 \\
\hline Kentucky L\&D & 15 & 37.01527 & -88.26543 & Tennessee & 22.4 \\
\hline Smithland L\&D & 40 & 37.166449 & -88.428196 & Ohio & 918.5 \\
\hline John T. Myers L\&D & 50 & 37.7949 & -87.99253 & Ohio & 846 \\
\hline Green River L\&D 1 & 15 & 37.85919 & -87.40863 & Green and Barren & 9.1 \\
\hline Newburgh L\&D & 15 & 37.9322316 & -87.373916 & Ohio & 776.1 \\
\hline Cannelton L\&D & 25 & 37.89969 & -86.70638 & Ohio & 720.7 \\
\hline McAlpine L\&D & 20 & 38.279045 & -85.79124 & Ohio & 606.8 \\
\hline *Louisville & 549 & 38.2549017 & -85.757235 & Ohio & 604 \\
\hline Markland L\&D & 13 & 38.774345 & -84.96612 & Ohio & 531.5 \\
\hline${ }^{*}$ Fort Thomas & 120 & 39.0630556 & -84.441389 & Ohio & 462.5 \\
\hline Meldahl L\&D & 15 & 38.79734 & -84.17253 & Ohio & 436.2 \\
\hline Greenup L\&D & 35 & 38.64795 & -82.86117 & Ohio & 341 \\
\hline${ }^{*}$ Huntington & 172 & 38.3944444 & -82.491389 & Ohio & 311.7 \\
\hline
\end{tabular}


ERDC/CHL CHETN-IX-57

June 2021

\begin{tabular}{|l|l|l|l|l|l||}
\hline Robert C Byrd L\&D & 15 & 38.6827783 & -82.184716 & Ohio & 279.2 \\
\hline Racine L\&D & 15 & 38.9183783 & -81.911266 & Ohio & 237.5 \\
\hline Belleville L\&D & 20 & 39.11696 & -81.74283 & Ohio & 203.9 \\
\hline Willow Island L\&D & 30 & 39.3602416 & -81.321566 & Ohio & 161.7 \\
\hline Hannibal L\&D & 5 & 39.66525 & -80.86585 & Ohio & 126.4 \\
\hline Pike Island L\&D & 25 & 40.1480183 & -80.701333 & Ohio & 84.2 \\
\hline $\begin{array}{l}\text { New Cumberland } \\
\text { L\&D }\end{array}$ & 25 & 40.5258133 & -80.62815 & Ohio & 54.4 \\
\hline Montgomery L\&D & 30 & 40.647975 & -80.385266 & Ohio & 31.7 \\
\hline Dashields L\&D & 25 & 40.54882 & -80.20663 & Ohio & 13.3 \\
\hline Emsworth L\&D & 25 & 40.505 & -80.08853 & Ohio & 6.2 \\
\hline *Pittsburgh & 460 & 40.4719444 & -79.994444 & Ohio & 0 \\
\hline \hline
\end{tabular}

*USCG NAIS site; all other sites USACE LOMA sites.

${ }^{* *} \mathrm{~L} \& \mathrm{D}=$ lock and dam

METHOD: The USACE eHydro program National Channel Framework (NCF) has divided the Ohio River into geospatially referenced polygons, known as reaches (USACE-eHydro 2020). The reaches are defined from bank to bank and are continuous for the length of the Ohio River. NCF reaches along inland waterways are generally, but not always, $1 \mathrm{mi}$ in length. This project developed a method for identifying which NCF reaches contain AIS position reports from the NAIS, including reaches with no coverage or partial coverage. AIS data were accessed via the USACE Automatic Identification System Analysis Package (AISAP) software (USACE-ERDC 2018). AISAP enables users to query AIS data from the USCG NAIS at different spatial-temporal scales and data sampling rates. This study requested data from calendar year 2018 for the Ohio River at a 5 min sampling rate interval. The returned AIS position reports were plotted in relation to the NCF reaches that make up the Ohio River using the ArcMap 10.7.1 software program (ESRI 2019). The number of position reports per reach was the output. The count of AIS position report points was normalized by dividing the number of points per reach by the reach's length, resulting in the metric position reports per reach per mile.

Vessel transit counts were acquired from the USACE Lock Performance Monitoring System (LPMS) records (USACE-IWR 2020). These counts were used to determine the minimum number of AIS points per reach expected during 2018. LPMS data are recorded by USACE personnel at lock sites, and each record includes the time of lock transit and vessel identity. Thus, LPMS data can be used to ground truth AIS data and any transit counts derived from AIS data. Recreational vessel lockages were excluded from LPMS records because the authors assumed they did not have AIS transmit capability. Records from neighboring locks were compared to determine each instance when a vessel passed from one lock to the next. This would count as one transit between the locks, and thus, AIS records - if complete - should show the presence of that vessel in any NCF reach located between those locks. This process was repeated for all neighboring locks, for all records, to determine the minimum number of transits per reach. For reaches that did not fall between two locks, which were reaches upstream of Emsworth Lock \& Dam and also reaches downstream of the Olmstead Locks and Dam, the number of transits per lock for the nearest lock was used as a proxy for the minimum expected transit count.

To translate the number of transits to the expected number of AIS data points, some assumptions about vessel speed were made. AIS position reports include speed over ground; the fastest vessel speed recorded in the 2018 data was $8 \mathrm{kn}$. At $8 \mathrm{kn}$, a vessel can travel $0.77 \mathrm{mi}$ in $5 \mathrm{~min}$. 
Therefore, it was assumed that a 5 min AIS data sampling rate, at least one AIS data point per mile per transit should be returned. Even for a vessel traveling at $10 \mathrm{kn}$, a data point should be received once every $5 \mathrm{~min}$ per mile as a vessel can travel $0.96 \mathrm{mi}$ in $5 \mathrm{~min}$. If a vessel were traveling $5 \mathrm{kn}$ or slower, than there should have been more than one data point per mile. This speed assumption contributes to the possibility of false positives for coverage because any vessel traveling under $5 \mathrm{kn}$ would contribute more AIS position reports per reach than expected. However, with this method, if a reach is showing as having less than $100 \%$ coverage, it is a true negative in terms of not having historical AIS position reports recorded for that reach.

RESULTS: The analysis was conducted for the $530 \mathrm{NCF}$ reaches that make up the 981 river miles of the Ohio River. The results shown in Table 3, indicate that 32 reaches have no coverage $(0 \%$ of the expected position reports are recorded for the corresponding NCF reach, highlighted in red), 98 reaches have low coverage ( $1 \%-25 \%$ of expected position reports, highlighted in orange), 37 reaches have limited coverage $(25 \%-50 \%$ of expected position reports, highlighted in yellow; $51 \%-75 \%$ of expected position reports, highlighted in light green), and 363 reaches have good coverage $(76 \%-$ $100 \%$ of expected position reports, highlighted in dark green). Within the table, reaches are listed from farthest upstream reach (CELRP_OH_LP_EMS_1), starting at Ohio River Mile 0, to farthest downstream reach (CELRL_OH_LD_CĀ_1), ending at Ohio River Mile 981, with the corresponding river mile boundaries for each reach. In Table 3 (pages 5, 6, 7, 8), when more than two consecutive reaches have $100 \%$ coverage, only the most upstream and downstream reaches with $100 \%$ coverage are listed; reaches in between are replaced by a row containing only ellipsis (...).

\section{Table 3. AIS coverage rate by Ohio River reach, 2018.}

\begin{tabular}{|c|c|c|c|}
\hline $\begin{array}{l}\text { Reach ID, USACE } \\
\text { National Channel } \\
\text { Framework }\end{array}$ & $\begin{array}{l}\text { Up- } \\
\text { stream } \\
\text { River } \\
\text { Mile }\end{array}$ & $\begin{array}{l}\text { Down- } \\
\text { stream } \\
\text { River } \\
\text { Mile }\end{array}$ & $\begin{array}{l}\text { AIS } \\
\text { Cover- } \\
\text { age } \\
\text { Rate } \\
(\%)\end{array}$ \\
\hline CELRP_OH_LP_EMS_1 & 0 & 1 & 100 \\
\hline \multicolumn{4}{|l|}{$\cdots$} \\
\hline CELRP_OH_LP_NC2_3 & 42 & 43 & 100 \\
\hline CELRP_OH_LP_NC2_4 & 43 & 44 & 52 \\
\hline CELRP_OH_LP_NC2_5 & 44 & 45 & 100 \\
\hline \multicolumn{4}{|l|}{$\ldots$} \\
\hline CELRP_OH_LP_PIK_10 & 63 & 64 & 100 \\
\hline CELRP_OH_LP_PIK_11 & 64 & 65 & 4 \\
\hline CELRP_OH_LP_PIK_12 & 65 & 66 & 1 \\
\hline CELRP_OH_LP_PIK_13 & 66 & 67 & 0 \\
\hline CELRP_OH_LP_PIK_14 & 67 & 68 & 6 \\
\hline CELRP_OH_LP_PIK_15 & 68 & 69 & 49 \\
\hline CELRP_OH_LP_PIK_16 & 69 & 70 & 100 \\
\hline \multicolumn{4}{|l|}{$\ldots$} \\
\hline CELRP_OH_LP_HAN_19 & 102 & 103 & 100 \\
\hline CELRP_OH_LP_HAN_20 & 103 & 104 & 1 \\
\hline CELRP_OH_LP_HAN_21 & 104 & 105 & 0 \\
\hline CELRP_OH_LP_HAN_22 & 105 & 106 & 1 \\
\hline CELRP_OH_LP_HAN_23 & 106 & 107 & 0 \\
\hline
\end{tabular}

\begin{tabular}{|c|c|c|c|}
\hline $\begin{array}{l}\text { Reach ID, USACE } \\
\text { National Channel } \\
\text { Framework }\end{array}$ & $\begin{array}{l}\text { Up- } \\
\text { stream } \\
\text { River } \\
\text { Mile }\end{array}$ & $\begin{array}{l}\text { Down- } \\
\text { stream } \\
\text { River } \\
\text { Mile }\end{array}$ & $\begin{array}{l}\text { AIS } \\
\text { Cover- } \\
\text { age } \\
\text { Rate } \\
(\%)\end{array}$ \\
\hline CELRH_OH_HD_RAO_19 & 220 & 221 & 3 \\
\hline CELRH_OH_HD_RAO_18 & 221 & 222 & 11 \\
\hline CELRH_OH_HD_RAO_17 & 222 & 223 & 1 \\
\hline CELRH_OH_HD_RAO_16 & 223 & 224 & 5 \\
\hline CELRH_OH_HD_RAO_15 & 224 & 225 & 1 \\
\hline CELRH_OH_HD_RAO_14 & 225 & 226 & 4 \\
\hline CELRH_OH_HD_RAO_13 & 226 & 227 & 22 \\
\hline CELRH_OH_HD_RAO_12 & 227 & 228 & 12 \\
\hline CELRH_OH_HD_RAO_11 & 228 & 229 & 3 \\
\hline CELRH_OH_HD_RAO_10 & 229 & 230 & 81 \\
\hline CELRH_OH_HD_RAO_9 & 230 & 231 & 29 \\
\hline CELRH_OH_HD_RAO_9 & 230 & 231 & 29 \\
\hline CELRH_OH_HD_RAO_8 & 231 & 232 & 100 \\
\hline \multicolumn{4}{|l|}{$\ldots$} \\
\hline CELRH_OH_HD_RCB_37 & 243 & 244 & 100 \\
\hline CELRH_OH_HD_RCB_36 & 244 & 245 & 7 \\
\hline CELRH_OH_HD_RCB_35 & 245 & 246 & 2 \\
\hline CELRH_OH_HD_RCB_34 & 246 & 247 & 2 \\
\hline CELRH_OH_HD_RCB_33 & 247 & 248 & 14 \\
\hline
\end{tabular}




\section{Table 3. AIS coverage rate by Ohio River reach, 2018.}

\begin{tabular}{|c|c|c|c|c|c|c|c|}
\hline $\begin{array}{l}\text { Reach ID, USACE } \\
\text { National Channel } \\
\text { Framework }\end{array}$ & $\begin{array}{l}\text { Up- } \\
\text { stream } \\
\text { River } \\
\text { Mile }\end{array}$ & $\begin{array}{l}\text { Down- } \\
\text { stream } \\
\text { River } \\
\text { Mile }\end{array}$ & $\begin{array}{l}\text { AIS } \\
\text { Cover- } \\
\text { age } \\
\text { Rate } \\
(\%)\end{array}$ & $\begin{array}{l}\text { Reach ID, USACE } \\
\text { National Channel } \\
\text { Framework }\end{array}$ & $\begin{array}{l}\text { Up- } \\
\text { stream } \\
\text { River } \\
\text { Mile }\end{array}$ & $\begin{array}{l}\text { Down- } \\
\text { stream } \\
\text { River } \\
\text { Mile }\end{array}$ & $\begin{array}{l}\text { AIS } \\
\text { Cover- } \\
\text { age } \\
\text { Rate } \\
(\%)\end{array}$ \\
\hline CELRP_OH_LP_HAN_24 & 107 & 108 & 0 & CELRH_OH_HD_RCB_32 & 248 & 249 & 12 \\
\hline CELRP_OH_LP_HAN_25 & 108 & 109 & 0 & CELRH_OH_HD_RCB_31 & 249 & 250 & 28 \\
\hline CELRP_OH_LP_HAN_26 & 109 & 110 & 0 & CELRH_OH_HD_RCB_30 & 250 & 251 & 61 \\
\hline CELRP_OH_LP_HAN_27 & 110 & 111 & 18 & CELRH_OH_HD_RCB_29 & 251 & 252 & 100 \\
\hline CELRP_OH_LP_HAN_28 & 111 & 112 & 4 & CELRH_OH_HD_RCB_28 & 252 & 253 & 48 \\
\hline CELRP_OH_LP_HAN_29 & 112 & 113 & 4 & CELRH_OH_HD_RCB_27 & 253 & 254 & 27 \\
\hline CELRP_OH_LP_HAN_30 & 113 & 114 & 1 & CELRH_OH_HD_RCB_26 & 254 & 255 & 100 \\
\hline CELRP_OH_LP_HAN_31 & 114 & 115 & 0 & \multicolumn{4}{|l|}{$\ldots$} \\
\hline CELRP_OH_LP_HAN_32 & 115 & 116 & 1 & CELRH_OH_HD_CAM_80 & 358 & 359 & 100 \\
\hline CELRP_OH_LP_HAN_33 & 116 & 117 & 66 & CELRH_OH_HD_CAM_79 & 359 & 360 & 39 \\
\hline CELRP_OH_LP_HAN_34 & 117 & 118 & 100 & CELRH_OH_HD_CAM_78 & 360 & 361 & 76 \\
\hline \multicolumn{4}{|l|}{$\ldots$} & CELRH_OH_HD_CAM_77 & 361 & 362 & 37 \\
\hline CELRH_OH_HD_WIO_30 & 133 & 134 & 100 & CELRH_OH_HD_CAM_76 & 362 & 363 & 95 \\
\hline CELRH_OH_HD_WIO_29 & 134 & 135 & 0 & CELRH_OH_HD_CAM_75 & 363 & 364 & 76 \\
\hline CELRH_OH_HD_WIO_29 & 134 & 135 & 0 & CELRH_OH_HD_CAM_74 & 364 & 365 & 74 \\
\hline CELRH_OH_HD_WIO_28 & 135 & 136 & 81 & CELRH_OH_HD_CAM_72 & 365 & 367 & 12 \\
\hline CELRH_OH_HD_WIO_27 & 136 & 137 & 88 & CELRH_OH_HD_CAM_73 & 365 & 366 & 64 \\
\hline CELRH_OH_HD_WIO_26 & 137 & 138 & 48 & CELRH_OH_HD_CAM_71 & 367 & 368 & 48 \\
\hline CELRH_OH_HD_WIO_25 & 138 & 139 & 29 & CELRH_OH_HD_CAM_70 & 368 & 369 & 55 \\
\hline CELRH_OH_HD_WIO_24 & 139 & 140 & 22 & CELRH_OH_HD_CAM_69 & 369 & 370 & 100 \\
\hline CELRH_OH_HD_WIO_23 & 140 & 141 & 7 & \multicolumn{4}{|l|}{$\ldots$} \\
\hline CELRH_OH_HD_WIO_22 & 141 & 142 & 4 & CELRH_OH_HD_CAM_66 & 372 & 373 & 100 \\
\hline CELRH_OH_HD_WIO_21 & 142 & 143 & 1 & CELRH_OH_HD_CAM_65 & 373 & 374 & 89 \\
\hline CELRH_OH_HD_WIO_20 & 143 & 144 & 1 & CELRH_OH_HD_CAM_64 & 374 & 375 & 29 \\
\hline CELRH_OH_HD_WIO_19 & 144 & 145 & 0 & CELRH_OH_HD_CAM_63 & 375 & 376 & 13 \\
\hline CELRH_OH_HD_WIO_18 & 145 & 146 & 0 & CELRH_OH_HD_CAM_62 & 376 & 377 & 11 \\
\hline CELRH_OH_HD_WIO_17 & 146 & 147 & 0 & CELRH_OH_HD_CAM_61 & 377 & 378 & 28 \\
\hline CELRH_OH_HD_WIO_16 & 147 & 148 & 1 & CELRH_OH_HD_CAM_60 & 378 & 379 & 11 \\
\hline CELRH_OH_HD_WIO_15 & 148 & 149 & 0 & CELRH_OH_HD_CAM_59 & 379 & 380 & 15 \\
\hline CELRH_OH_HD_WIO_14 & 149 & 150 & 0 & CELRH_OH_HD_CAM_58 & 380 & 381 & 25 \\
\hline CELRH_OH_HD_WIO_13 & 150 & 151 & 1 & CELRH_OH_HD_CAM_57 & 381 & 382 & 26 \\
\hline CELRH_OH_HD_WIO_12 & 151 & 152 & 0 & CELRH_OH_HD_CAM_56 & 382 & 383 & 16 \\
\hline CELRH_OH_HD_WIO_11 & 152 & 153 & 1 & CELRH_OH_HD_CAM_55 & 383 & 384 & 22 \\
\hline CELRH_OH_HD_WIO_10 & 153 & 154 & 0 & CELRH_OH_HD_CAM_54 & 384 & 385 & 38 \\
\hline CELRH_OH_HD_WIO_10 & 153 & 154 & 0 & CELRH_OH_HD_CAM_53 & 385 & 386 & 50 \\
\hline CELRH_OH_HD_WIO_9 & 154 & 155 & 0 & CELRH_OH_HD_CAM_52 & 386 & 387 & 36 \\
\hline CELRH_OH_HD_WIO_9 & 154 & 155 & 0 & CELRH_OH_HD_CAM_51 & 387 & 388 & 36 \\
\hline CELRH_OH_HD_WIO_8 & 155 & 156 & 1 & CELRH_OH_HD_CAM_50 & 388 & 389 & 54 \\
\hline CELRH_OH_HD_WIO_7 & 156 & 157 & 12 & CELRH_OH_HD_CAM_49 & 389 & 390 & 27 \\
\hline
\end{tabular}


Table 3. AIS coverage rate by Ohio River reach, 2018.

\begin{tabular}{|c|c|c|c|c|c|c|c|}
\hline $\begin{array}{l}\text { Reach ID, USACE } \\
\text { National Channel } \\
\text { Framework }\end{array}$ & $\begin{array}{l}\text { Up- } \\
\text { stream } \\
\text { River } \\
\text { Mile }\end{array}$ & $\begin{array}{l}\text { Down- } \\
\text { stream } \\
\text { River } \\
\text { Mile }\end{array}$ & $\begin{array}{l}\text { AIS } \\
\text { Cover- } \\
\text { age } \\
\text { Rate } \\
(\%)\end{array}$ & $\begin{array}{l}\text { Reach ID, USACE } \\
\text { National Channel } \\
\text { Framework }\end{array}$ & $\begin{array}{l}\text { Up- } \\
\text { stream } \\
\text { River } \\
\text { Mile }\end{array}$ & $\begin{array}{l}\text { Down- } \\
\text { stream } \\
\text { River } \\
\text { Mile }\end{array}$ & $\begin{array}{l}\text { AIS } \\
\text { Cover- } \\
\text { age } \\
\text { Rate } \\
(\%)\end{array}$ \\
\hline CELRH_OH_HD_WIO_6 & 157 & 158 & 100 & CELRH_OH_HD_CAM_48 & 390 & 391 & 21 \\
\hline \multicolumn{4}{|l|}{$\ldots$} & CELRH_OH_HD_CAM_47 & 391 & 392 & 38 \\
\hline CELRH_OH_HD_BEL_40 & 165 & 166 & 100 & CELRH_OH_HD_CAM_46 & 392 & 393 & 25 \\
\hline CELRH_OH_HD_BEL_39 & 166 & 167 & 3 & CELRH_OH_HD_CAM_45 & 393 & 394 & 14 \\
\hline CELRH_OH_HD_BEL_38 & 167 & 168 & 0 & CELRH_OH_HD_CAM_44 & 394 & 395 & 4 \\
\hline CELRH_OH_HD_BEL_37 & 168 & 169 & 0 & CELRH_OH_HD_CAM_43 & 395 & 396 & 2 \\
\hline CELRH_OH_HD_BEL_36 & 169 & 170 & 0 & CELRH_OH_HD_CAM_42 & 396 & 397 & 2 \\
\hline CELRH_OH_HD_BEL_36 & 169 & 170 & 0 & CELRH_OH_HD_CAM_41 & 397 & 398 & 1 \\
\hline CELRH_OH_HD_BEL_35 & 170 & 171 & 0 & CELRH_OH_HD_CAM_40 & 398 & 399 & 2 \\
\hline CELRH_OH_HD_BEL_35 & 170 & 171 & 0 & CELRH_OH_HD_CAM_39 & 399 & 400 & 1 \\
\hline CELRH_OH_HD_BEL_34 & 171 & 172 & 1 & CELRH_OH_HD_CAM_38 & 400 & 401 & 1 \\
\hline CELRH_OH_HD_BEL_33 & 172 & 173 & 6 & CELRH_OH_HD_CAM_37 & 401 & 402 & 1 \\
\hline CELRH_OH_HD_BEL_32 & 173 & 174 & 10 & CELRH_OH_HD_CAM_36 & 402 & 403 & 38 \\
\hline CELRH_OH_HD_BEL_31 & 174 & 175 & 7 & CELRH_OH_HD_CAM_35 & 403 & 404 & 100 \\
\hline CELRH_OH_HD_BEL_30 & 175 & 176 & 3 & \multicolumn{4}{|l|}{$\ldots$} \\
\hline CELRH_OH_HD_BEL_29 & 176 & 177 & 0 & CELRH_OH_HD_CAM_33 & 405 & 406 & 100 \\
\hline CELRH_OH_HD_BEL_29 & 176 & 177 & 0 & CELRH_OH_HD_CAM_32 & 406 & 407 & 39 \\
\hline CELRH_OH_HD_BEL_28 & 177 & 178 & 6 & CELRH_OH_HD_CAM_31 & 407 & 408 & 21 \\
\hline CELRH_OH_HD_BEL_27 & 178 & 179 & 32 & CELRH_OH_HD_CAM_30 & 408 & 409 & 17 \\
\hline CELRH_OH_HD_BEL_26 & 179 & 180 & 1 & CELRH_OH_HD_CAM_29 & 409 & 410 & 3 \\
\hline CELRH_OH_HD_BEL_25 & 180 & 181 & 2 & CELRH_OH_HD_CAM_28 & 410 & 411 & 2 \\
\hline CELRH_OH_HD_BEL_24 & 181 & 182 & 1 & CELRH_OH_HD_CAM_27 & 411 & 412 & 26 \\
\hline CELRH_OH_HD_BEL_23 & 182 & 183 & 1 & CELRH_OH_HD_CAM_26 & 412 & 413 & 100 \\
\hline CELRH_OH_HD_BEL_22 & 183 & 184 & 1 & \multicolumn{4}{|l|}{$\ldots$} \\
\hline CELRH_OH_HD_BEL_21 & 184 & 185 & 2 & CELRH_OH_HD_CAM_24 & 414 & 415 & 100 \\
\hline CELRH_OH_HD_BEL_20 & 185 & 186 & 1 & CELRH_OH_HD_CAM_23 & 415 & 416 & 5 \\
\hline CELRH_OH_HD_BEL_19 & 186 & 187 & 1 & CELRH_OH_HD_CAM_22 & 416 & 417 & 16 \\
\hline CELRH_OH_HD_BEL_18 & 187 & 188 & 0 & CELRH_OH_HD_CAM_21 & 417 & 418 & 100 \\
\hline CELRH_OH_HD_BEL_18 & 187 & 188 & 0 & \multicolumn{4}{|l|}{$\ldots$} \\
\hline CELRH_OH_HD_BEL_17 & 188 & 189 & 0 & CELRL_OH_LD_MCA_6 & 540 & 544 & 100 \\
\hline CELRH_OH_HD_BEL_17 & 188 & 189 & 0 & CELRL_OH_LD_MCA_5 & 544 & 556 & 38 \\
\hline CELRH_OH_HD_BEL_16 & 189 & 190 & 7 & CELRL_OH_LD_MCA_4 & 556 & 565 & 100 \\
\hline CELRH_OH_HD_BEL_15 & 190 & 191 & 5 & \multicolumn{4}{|l|}{$\ldots$} \\
\hline CELRH_OH_HD_BEL_14 & 191 & 192 & 5 & CELRL_OH_LD_CAN_7 & 625 & 639 & 100 \\
\hline CELRH_OH_HD_BEL_13 & 192 & 193 & 1 & CELRL_OH_LD_CAN_6 & 639 & 659 & 4 \\
\hline CELRH_OH_HD_BEL_12 & 193 & 194 & 2 & CELRL_OH_LD_CAN_5 & 659 & 664 & 4 \\
\hline CELRH_OH_HD_BEL_11 & 194 & 195 & 2 & CELRL_OH_LD_CAN_4 & 664 & 680 & 11 \\
\hline CELRH_OH_HD_BEL_10 & 195 & 196 & 1 & CELRL_OH_LD_CAN_3 & 680 & 700 & 23 \\
\hline CELRH_OH_HD_BEL_9 & 196 & 197 & 13 & CELRL_OH_LD_CAN_2 & 700 & 720 & 100 \\
\hline
\end{tabular}




\begin{tabular}{|c|c|c|c|c|c|c|c|}
\hline $\begin{array}{l}\text { Reach ID, USACE } \\
\text { National Channel } \\
\text { Framework }\end{array}$ & $\begin{array}{l}\text { Up- } \\
\text { stream } \\
\text { River } \\
\text { Mile }\end{array}$ & $\begin{array}{l}\text { Down- } \\
\text { stream } \\
\text { River } \\
\text { Mile }\end{array}$ & $\begin{array}{l}\text { AIS } \\
\text { Cover- } \\
\text { age } \\
\text { Rate } \\
(\%)\end{array}$ & $\begin{array}{l}\text { Reach ID, USACE } \\
\text { National Channel } \\
\text { Framework }\end{array}$ & $\begin{array}{l}\text { Up- } \\
\text { stream } \\
\text { River } \\
\text { Mile }\end{array}$ & $\begin{array}{l}\text { Down- } \\
\text { stream } \\
\text { River } \\
\text { Mile }\end{array}$ & $\begin{array}{l}\text { AIS } \\
\text { Cover- } \\
\text { age } \\
\text { Rate } \\
\text { (\%) }\end{array}$ \\
\hline CELRH_OH_HD_BEL_8 & 197 & 198 & 97 & \multicolumn{4}{|l|}{$\ldots$} \\
\hline CELRH_OH_HD_BEL_7 & 198 & 199 & 79 & CELRL_OH_LD_NBG_10 & 731 & 733 & 100 \\
\hline CELRH_OH_HD_BEL_6 & 199 & 200 & 100 & CELRL_OH_LD_NBG_9 & 733 & 738.5 & 32 \\
\hline \multicolumn{4}{|l|}{$\ldots$} & CELRL_OH_LD_NBG_8 & 738.5 & 741.2 & 67 \\
\hline CELRH_OH_HD_RAO_31 & 208 & 209 & 100 & CELRL_OH_LD_NBG_7 & 741.2 & 745 & 100 \\
\hline CELRH_OH_HD_RAO_30 & 209 & 210 & 59 & \multicolumn{4}{|l|}{$\ldots$} \\
\hline CELRH_OH_HD_RAO_29 & 210 & 211 & 0 & CELRL_OH_LD_SMT_11 & 862 & 865 & 100 \\
\hline CELRH_OH_HD_RAO_28 & 211 & 212 & 1 & CELRL_OH_LD_SMT_10 & 865 & 868.6 & 60 \\
\hline CELRH_OH_HD_RAO_27 & 212 & 213 & 44 & CELRL_OH_LD_SMT_9 & 868.6 & 872.8 & 17 \\
\hline CELRH_OH_HD_RAO_26 & 213 & 214 & 95 & CELRL_OH_LD_SMT_8 & 872.8 & 877.1 & 3 \\
\hline CELRH_OH_HD_RAO_25 & 214 & 215 & 8 & CELRL_OH_LD_SMT_7 & 877.1 & 884 & 2 \\
\hline CELRH_OH_HD_RAO_24 & 215 & 216 & 16 & CELRL_OH_LD_SMT_6 & 884 & 891 & 5 \\
\hline CELRH_OH_HD_RAO_23 & 216 & 217 & 10 & CELRL_OH_LD_SMT_5 & 891 & 898 & 7 \\
\hline CELRH_OH_HD_RAO_22 & 217 & 218 & 23 & CELRL_OH_LD_SMT_4 & 898 & 903 & 100 \\
\hline CELRH_OH_HD_RAO_21 & 218 & 219 & 12 & \multicolumn{4}{|l|}{$\ldots$} \\
\hline CELRH_OH_HD_RAO_20 & 219 & 220 & 3 & CELRL_OH_LD_CAI_1 & 979.5 & 981 & 100 \\
\hline
\end{tabular}

The presence or absence of AIS signals within an NCF reach appears dependent upon the terrain, rather than a predictable distance from an AIS site. Figure 2, below, shows a map of expected vs. received position reports for the reaches covering River Miles 0 to 74. Figure 2 shows that from River Mile 0 through 64, there is good coverage, but then coverage declines across River Miles 65 through 69 , which corresponds to a sharp bend in the river valley. 


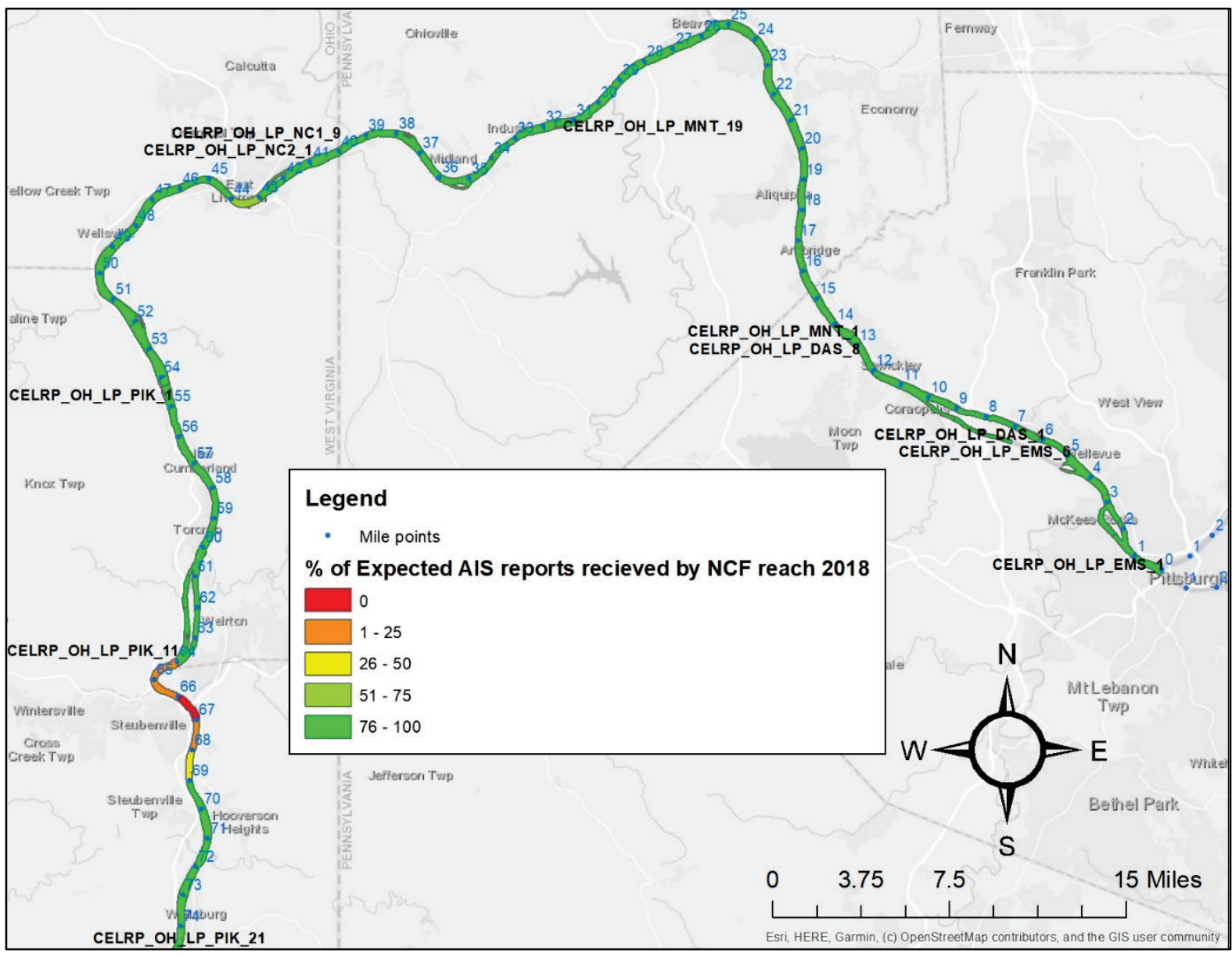

Figure 2. Map of expected vs. received AIS position reports for reaches covering Ohio River Miles 0 to 74 in year 2018 .

One drawback to this method is the possibility of false positives, where a reach may appear to have full spatial coverage along its entire length when in fact it does not. Despite this limitation, the method described here identifies reaches that have no coverage and are thus potential candidates for improving coverage. There are several ways to fill coverage gaps, including improving existing sites, installing new sites, using repeaters, and possibly purchasing data from a commercial source. Users of historic Ohio River AIS data should be alerted to the presence of these data gaps, which may limit the scope of other waterway investigations. Future work in the area of AIS coverage analysis may include the following approaches: removing AIS reports with zero speed to look at only moving vessels; examining years beyond 2018; utilizing a different sampling rate; or developing measures that better reveal the extent of partial coverage. In addition, the coverage analysis can be correlated to characteristics of the surrounding area and closest AIS site locations to determine any dependencies, such as with antenna height, AIS site features, distance to nearest site, and topography. Finally, this method can be applied to additional navigable rivers to expand the sample size and also to inform waterway stakeholders.

ADDITIONAL INFORMATION: This CHETN was prepared by Patricia K. DiJoseph, Patricia.K.DiJoseph@usace.army.mil, Brian J. Tetreault, Brian.J.Tetreault@usace.army.mil, and Marin M. Kress, Marin.M.Kress@usace.army.mil, (ORCiD https://orcid.org/0000-0002-5835-5686) Coastal and Hydraulics Laboratory, US Army Engineer Research and Development Center. Sincere 
appreciation is expressed to the AIS data team members (including L. Whitlow, B. Towne, J. Kilroy, R. Akers, S. Herrle, and K. N. Mitchell) and the USCG NAIS program. The study was funded by the USACE Navigation Systems Research Program. This CHETN should be cited as follows:

DiJoseph, P. K., B. J. Tetreault, and M. M. Kress. 2021. AIS Data Case Study: Identifying AIS Coverage Gaps on the Ohio River in CY2018. ERDC/CHL CHETN-IX-57. Vicksburg, MS: US Army Engineer Research and Development Center. http://dx.doi.org/10.21079/11681/40886.

\section{REFERENCES}

Dijoseph, P. K., K. N. Mitchell, B. J. Tetreault, and J. Marshall. 2019. Inland Marine Transportation System Travel Time Atlas via Automatic Identification System (AIS) Data: Ohio River, Upper Mississippi River, and Illinois River. ERDC/CHL TR-19-15. Vicksburg, MS: US Army Engineer Research and Development Center. https://apps.dtic.mil/sti/citations/AD1080408

ESRI. 2019. ArcMap 10.7.1. [software program]. Redlands, CA.

IALA (International Association of Marine Aids to Navigation and Lighthouse Authorities). 2008. Establishment of AIS as an Aid to Navigation: Report ID 1062. https://www.iala-aism.org/product/establishment-of-ais-as-an-aid-tonavigation-1062/

IEC (International Electrotechnical Commission). 2001. EC 61993-2: 2001-12 Maritime Navigation and Radiocommunication Equipment and Systems - Automatic Identification Systems (AIS) - Part 2: Class A Shipborne Equipment of the Universal Automatic Identification System (AIS) - Operational and Performance Requirements, Methods of Test and Required Test Results. (Technical standards report). https://www.iec.ch/index.htm

ITU-R (International Telecommunications Union - Radiocommunication Sector). 2014. Recommendation ITU-R M.1371: Technical Characteristics for an Automatic Identification System Using Time Division Multiple Access in the VHF Maritime Mobile Frequency Band. Report. https://www.itu.int/rec/R-REC-M.1371/en

Kress, M. M., B. J. Tetreault, K. N. Mitchell, M. Balazik, and M. C. Booton 2020. AIS Data: Real-Time Operation Support, Incident Investigations, and Waterway Use Analysis. ERDC/CHL CHETN-IX-53. Vicksburg, MS: US Army Engineer Research and Development Center. http://dx.doi.org/10.21079/11681/36395

Mitchell, K. N., and B. N. Scully. 2014. "Waterway Performance Monitoring via Automatic Identification System (AIS) Data." Transportation Research Record: Journal of Transportation 2426(1): 20-26. https://doi.org/10.3141/2426-03

Mitchell, K. N., P. K. DiJoseph, B. N. Scully, and M. M. Kress. 2020. Towing Vessel Delays and Barge Lane Navigability along the Houston Ship Channel, Texas. ERDC/CHL TR-20-1. Vicksburg, MS: US Army Engineer Research and Development Center.

PIANC (The World Association for Waterborne Transport Infrastructure). 2019. Guidelines and Recommendations for River Information Services. InCom Working Group Report Number 125/I-2019. https://www.pianc.org/publications/inland-navigation-commission/wg125-1

Scully, B., and K. N. Mitchell. 2015. Archival Automatic Identification System (AIS) Data for Navigation Project Performance Evaluation. ERDC/CHL CHETN-IX-40. Vicksburg, MS: US Army Engineer Research and Development Center. http://hdl.handle.net/11681/2066

Tabbert, C., J. Vest, A. Rhoads, D. Myers, T. Lauth, E. Brauer, J. Wallace, D. Gordon, and M. Kress. 2020. AIS Data Case Study: St. Louis Area Commercial Vessel Fleeting Activity and Potential River Training Structures. ERDC/CHL CHETN-IX-52. Vicksburg, MS: US Army Engineer Research and Development Center. http://dx.doi.org/10.21079/11681/36334 
USACE-eHydro (US Army Corps of Engineers-eHydro). 2020. National Channel Framework [shapefile database download]. US Army Corps of Engineers, Mobile, AL.

https://geospatial-usace.opendata.arcgis.com/datasets/9227967a2748410983352b501c0c7b39

USACE-ERDC (US Army Corps of Engineers, US Army Engineer Research and Development Center). 2018. AIS Analysis Package (AISAP). Software package. https://ais-portal.usace.army.mil

USCG (US Coast Guard). 2018. Navigation Center: Automatic Identification System Overview. http://www.navcen.uscg.gov/?pageName=AISmain

USACE-IWR (US Army Corps of Engineers-Institute for Water Resources) 2020. Lock Performance Monitoring System Public Web-Home. Washington, DC. Accessed 17 December 2020.

https://corpslocks.usace.army.mil/lpwb/f?p=121:1:1

US Code of Regulations. 2019. Title 33-Navigation and Navigable Waters. "Part 164-Navigation Safety Regulations." 33 C.F.R. § 164.46. Washington, DC. https://www.govinfo.gov/content/pkg/CFR-2019-title33-vol2/xml/CFR-2019title33-vol2-part164.xml

WCSC (USACE-Waterborne Commerce Statistics Center). 2021. Ports and Waterways Webtool: 2018 - Ohio River (Waterway). Washington, DC. Accessed 21 February.

http://cwbi-ndc-nav.s3-website-us-east-1.amazonaws.com/files/wcsc/webpub/\#/report-

landing/year/2018/region/2/location/2078

NOTE: The contents of this technical note are not to be used for advertising, publication or promotional purposes. Citation of trade names does not constitute an official 